\title{
Dominant gut Prevotella copri in gastrectomised non-obese diabetic Goto-Kakizaki rats improves glucose homeostasis through enhanced FXR signalling
}

\author{
Noémie Péan ${ }^{1}$ - Aurelie Le Lay ${ }^{1}$ • Francois Brial ${ }^{1}$ - Jessica Wasserscheid ${ }^{2}$ - Claude Rouch ${ }^{3}$ - Mylène Vincent ${ }^{3}$. \\ Antonis Myridakis ${ }^{4} \cdot$ Lyamine Hedjazi $^{5}$ - Marc-Emmanuel Dumas ${ }^{4}$. Elin Grundberg ${ }^{2} \cdot$ Mark Lathrop $^{2}$. \\ Christophe Magnan ${ }^{3} \cdot$ Ken Dewar $^{2}$ - Dominique Gauguier ${ }^{1,2}$
}

Received: 30 October 2019 / Accepted: 4 February 2020/Published online: 16 March 2020

(C) The Author(s) 2020

\begin{abstract}
Aims/hypothesis Drug and surgical-based therapies in type 2 diabetes are associated with altered gut microbiota architecture. Here we investigated the role of the gut microbiome in improved glucose homeostasis following bariatric surgery.

Methods We carried out gut microbiome analyses in gastrectomised (by vertical sleeve gastrectomy [VSG]) rats of the Goto-Kakizaki (GK) non-obese model of spontaneously occurring type 2 diabetes, followed by physiological studies in the GK rat.

Results VSG in the GK rat led to permanent improvement of glucose tolerance associated with minor changes in the gut microbiome, mostly characterised by significant enrichment of caecal Prevotella copri. Gut microbiota enrichment with P. copri in GK rats through permissive antibiotic treatment, inoculation of gut microbiota isolated from gastrectomised GK rats, and direct inoculation of $P$. copri, resulted in significant improvement of glucose tolerance, independent of changes in body weight. Plasma bile acids were increased in GK rats following inoculation with $P$. copri and $P$. copri-enriched microbiota from VSG-treated rats; the inoculated GK rats then showed increased liver glycogen and upregulated expression of Fxr (also known as Nr1h4), Srebflc, Chrebp (also known as Mlxipl) and Illo and downregulated expression of Cyp7a1.

Conclusions Our data underline the impact of intestinal $P$. copri on improved glucose homeostasis through enhanced bile acid metabolism and farnesoid X receptor (FXR) signalling, which may represent a promising opportunity for novel type 2 diabetes therapeutics.
\end{abstract}

Keywords $16 \mathrm{~S}$ rDNA $\cdot$ Bile acids $\cdot$ Goto-Kakizaki rat $\cdot$ Microbiome $\cdot$ Type 2 diabetes

Noémie Péan, Aurelie Le Lay and Francois Brial are joint first authors.

Electronic supplementary material The online version of this article (https://doi.org/10.1007/s00125-020-05122-7) contains peer-reviewed but unedited supplementary material, which is available to authorised users.

Ken Dewar

ken.dewar@mcgill.ca

Dominique Gauguier

dominique.gauguier@inserm.fr

1 Inserm UMR 1124, Université de Paris, 45 rue des Saint-Pères, 75006 Paris, France

2 McGill University and Genome Quebec Innovation Centre, 740 Doctor Penfield Avenue, Montreal, QC H3A 0G1, Canada
3 Unit of Functional and Adaptive Biology, UMR 8251, CNRS, Université de Paris, 4 rue Marie Andrée Lagroua Weill-Halle, Paris, France

4 Section of Biomolecular Medicine, Division of Computational and Systems Medicine, Department of Surgery and Cancer, Faculty of Medicine, Imperial College London, London, UK

5 Beemetrix SAS, Massy, France 


\section{Research in context}

\section{What is already known about this subject?}

- Bariatric surgery in spontaneously diabetic rats of the Goto-Kakizaki (GK) strain reduces glucose intolerance

- Intestinal Prevotella copri induces changes in host's glucose homeostasis

- Bariatric surgery is associated with changes in bile acids and liver farnesoid X receptor (FXR) expression

What is the key question?

- What are the mechanisms mediated by the intestinal microbiota that contribute to improved glucose homeostasis following bariatric surgery?

What are the new findings?

- Bariatric surgery in diabetic GK rats alters gut microbiota ecology and increases intestinal $P$. copri levels

- Gut microbiota enriched in P. copri improves glucose tolerance in diabetic GK rats

- $\quad$ Enriched intestinal P. copri in diabetic GK rats stimulates bile acids and liver FXR expression

How might this impact on clinical practice in the foreseeable future?

- $\quad$. copri is a candidate for the development of probiotic-based therapeutic approaches in diabetes

\author{
Abbreviations \\ CYP7A1 Cholesterol 7 $\alpha$-hydroxylase \\ FXR Farnesoid X receptor \\ GK Goto-Kakizaki \\ VSG Vertical sleeve gastrectomy
}

\section{Introduction}

The gastrointestinal microbiota is a complex system of commensal bacteria which plays crucial roles in host metabolism [1] and maturation of the immune system [2]. Its architecture adapts to disease conditions [3], including obesity and type 2 diabetes [4, 5], and to diabetes therapies, such as drug treatment and bariatric surgery $[6,7]$. Bariatric surgery techniques designed to induce weight loss in obese patients [8] are also associated with improved glucose homeostasis and even diabetes remission [9], but the underlying biological processes remain poorly understood. Alterations in the gut microbiome are proposed to mediate weight loss and improve glucose homeostasis following bariatric surgery [7].

Inbred preclinical models maintained in conditions limiting inter-individual phenotypic variability are powerful experimental systems to assessing functional relationships between gut microbiota and host physiology. The Goto-Kakizaki (GK) rat is an inbred model of spontaneous diabetes obtained by selective enrichment of naturally occurring genetic polymorphisms [10] resulting in multifaceted pathological features relevant to type 2 diabetes in the absence of obesity. Even though diabetescausing genes have been mapped to the GK genome (reviewed in [11]) [12], bariatric surgery improves glycaemic control in this strain [13-15] through unknown mechanisms.

To disentangle relationships between gastrectomy and improved glucose homeostasis, we carried out a series of metagenomic and physiological experiments in the GK rat in order to test associations between Prevotella copri, a dominant bacterial strain stimulated by vertical sleeve gastrectomy (VSG), the metabolism of bile acids and farnesoid $\mathrm{X}$ receptor (FXR) signalling. These data bring new insights into the impact of gut microbiota on glycaemic control associated with enhanced bile acid metabolism and FXR signalling.

\section{Methods}

For detailed Methods, please refer to the electronic supplementary material (ESM).

Animals Goto-Kakizaki (GK/Ox) rats bred in the Inserm UMR 1124 laboratory were maintained in a controlled environment ( $12 \mathrm{~h}$ dark-light cycles $22-24^{\circ} \mathrm{C}$; humidity $50-60 \%$ ). They had ad libitum access to water and standard chow (SAFE, Augy, France). Animals from the experimental and control groups were housed in different cages to avoid unintentional microbiota transplants via coprophagy. GK rats were used in a series of experiments outlined in Fig. 1. Procedures were reviewed by the University Ethics Committee in Animal Experiment and delivered by the French ministry of research under the licence 4231201602231507187. 


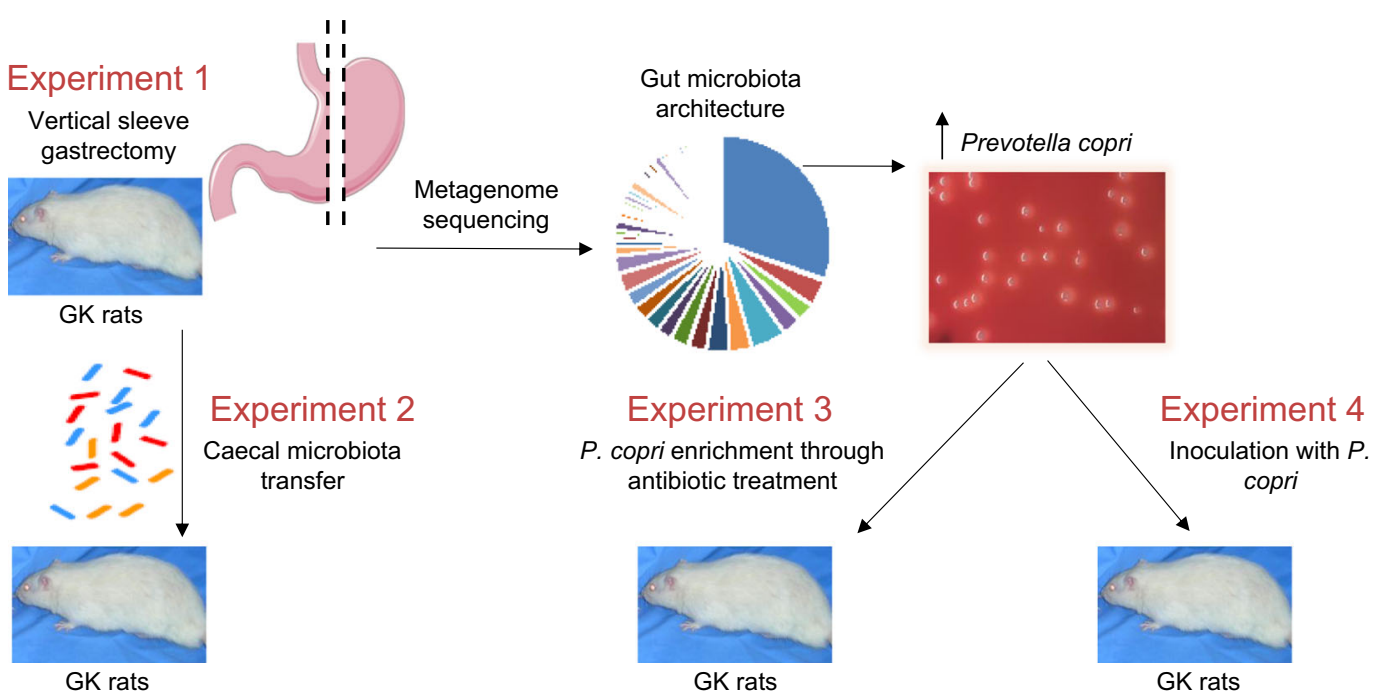

Fig. 1 Outline of experimental design

VSG VSG was performed in 16-week-old male GK rats. The lateral $80 \%$ of the stomach was excised with a cutter (TLC55, Ethicon, Issy, France). Control GK rats were sham operated by application of pressure between the oesophageal sphincter and the pylorus. Rats were killed 13 weeks after surgery. Caecum and colon contents were harvested, quick-frozen and stored at $-80^{\circ} \mathrm{C}$.

Pair-feeding studies Thirty days after surgery, sham rats were pair-fed to match the food intake of free-fed VSG GK rats. Blood glucose was determined using an Accu-Check, Performa (Roche Diagnostics, Meylan, France).

Gut microbiota transfer Five month old male GK rats were treated orally with Inexium (Omeprazole, AstraZeneca, Courbevoie, France) and Moviprep (Norgine, RueilMalmaison, France) [16, 17], and inoculated with caecal microbiota from VSG-treated or sham-operated GK rats.

Enrichment of gut microbiota with $P$. copri Five-month-old male GK rats were given vancomycin $(0.5 \mathrm{~g} / \mathrm{l})$ and kanamycin ( $1 \mathrm{~g} / \mathrm{l})$ for 10 days. GK controls remained in antibiotic-free conditions.

P. copri supplementation Five-month-old male GK rats were given vancomycin $(0.5 \mathrm{~g} / \mathrm{l})$, neomycin $(1 \mathrm{~g} / \mathrm{l})$, metronidazole (1 g/l) and ampicillin (1 g/l) over 10 days to wipe out gut bacteria and facilitate $P$. copri colonisation. Then rats were inoculated with single gavage of $5 \times 10^{8}$ CFU $P$. copri (DSM18205, DSMZ, Braunschweig, Germany) or heatkilled $P$. copri in controls.

Glucose tolerance tests and sample collection and analysis OGTTs (Experiment 1 in Fig. 1) and IPGTTs (Experiments 2, 3 and 4 in Fig. 1) were performed in conscious overnight fasted rats. Blood collected from the tail vein before administration of glucose ( $1 \mathrm{~g} / \mathrm{kg}$ body weight) and sequentially afterwards was used to determine blood glucose (Roche Diagnostics) and insulin levels (Mercodia, Uppsala, Sweden).

Three days later, overnight fasted rats were killed. Plasma, adipose tissue, liver and caecal content were quick-frozen and stored at $-80^{\circ} \mathrm{C}$. Colorimetric assays were used to determine triacylglycerol (ab65336; Abcam, Paris, France) and glycogen (Sigma-Aldrich, Saint-Quentin, France) content.

Metagenome sequencing Bacterial DNA was prepared from caecal and colon samples (DNAStool mini kit, QIAGEN, Courtaboeuf, France). Whole-genome shotgun sequencing was performed on Illumina HiSeq 2000 (Illumina, San Diego, CA, USA). The sequence motifs corresponding to a 55 nucleotide segment of the bacterial 16S rRNA variable 1-3 region were used to estimate sample biodiversity.

Quantitative PCR P. copri enrichment was assessed by quantitative PCR of caecum bacterial DNA. Preparation of liver and adipose tissue RNA and quantitative RT-PCR were performed as described [18]. Oligonucleotide sequences are given in ESM Table 1.

Quantitative analysis of plasma bile acids Bile acids were quantified as described [19] using an ACQUITY BEH C8 column and a Xevo TQ-S mass spectrometer (both from Waters, Manchester, UK).

Statistical analyses $\mathrm{R}$ packages were used to assess differences in the frequency of $16 \mathrm{~S}$ rDNA motifs. $p$ values were corrected for multiple testing using the Benjamini-Hochberg method [20]. Blood glucose and insulin data during the glucose tolerance tests were analysed with the Kruskal-Wallis test. Nonparametric Mann-Whitney $U$ tests were used to analyse physiological phenotypes, bile acid data and gene expression. 


\section{Results}

\section{Gastrectomised GK rats exhibit improved glycaemic control} To verify the effect of bariatric surgery on glycaemic control in the GK rats [13-15], we performed VSG in our GK colony (Experiment 1 in Fig. 1). VSG induced an immediate reduction in body weight (Fig. 2a) and food intake (ESM Fig. 1) and decreased glucose levels (Fig. 2b) when compared with shamoperated GK rats. Gastrectomised rats regained body weight similar to that of controls between 4 and 6 weeks post surgery, but blood glucose remained significantly reduced over the 12 week period following VSG (Fig. 2b), when OGTTs were performed.

Due to extensive development of intra-abdominal connective tissue secondary to VSG, OGTTs were preferred to IPGTTs. Glucose tolerance was similar in GK rats before VSG and sham-operated GK, thus demonstrating that glucose homeostasis was not affected by sham operation (Fig. 2c). VSG resulted in improved glucose tolerance (Fig. 2c), assessed by the significant reduction of both cumulative glucose levels during the OGTT (Fig. 2d) and the $\Delta$ G parameter (Fig. 2e) when compared with sham GK rats.
Gastrectomised GK rats exhibited elevated basal insulin levels and enhanced glucose-induced insulin secretion when compared with controls (ESM Fig. 2a).

Inoculation of GK rats with gut microbiota from gastrectomised GK rats improves glucose tolerance To test whether the gut microbiota may mediate the metabolic effects of VSG, we inoculated GK rats with the caecal microbiota from gastrectomised or sham-operated GK rats (Experiment 2 in Fig. 1). Body weight was affected neither by antibiotic treatment prior to microbiota transplantation nor by gut microbiota transfer (Fig. 2f). Blood glucose was significantly reduced from 60 min after the glucose injection until the end of the IPGTT in rats inoculated with the microbiota from VSGtreated rats (Fig. 2g), which resulted in a significant reduction in both cumulative glucose levels (Fig. $2 \mathrm{~h}$ ) and the $\Delta \mathrm{G}$ parameter (Fig. 2i) when compared with GK rats inoculated with the microbiota from sham-operated animals. There were no differences in insulin secretion in the two groups (ESM Fig. 2b). These data demonstrate that gut microbiota changes induced by VSG in diabetic GK rats contribute to improving glucose tolerance when transplanted to diabetic recipients.
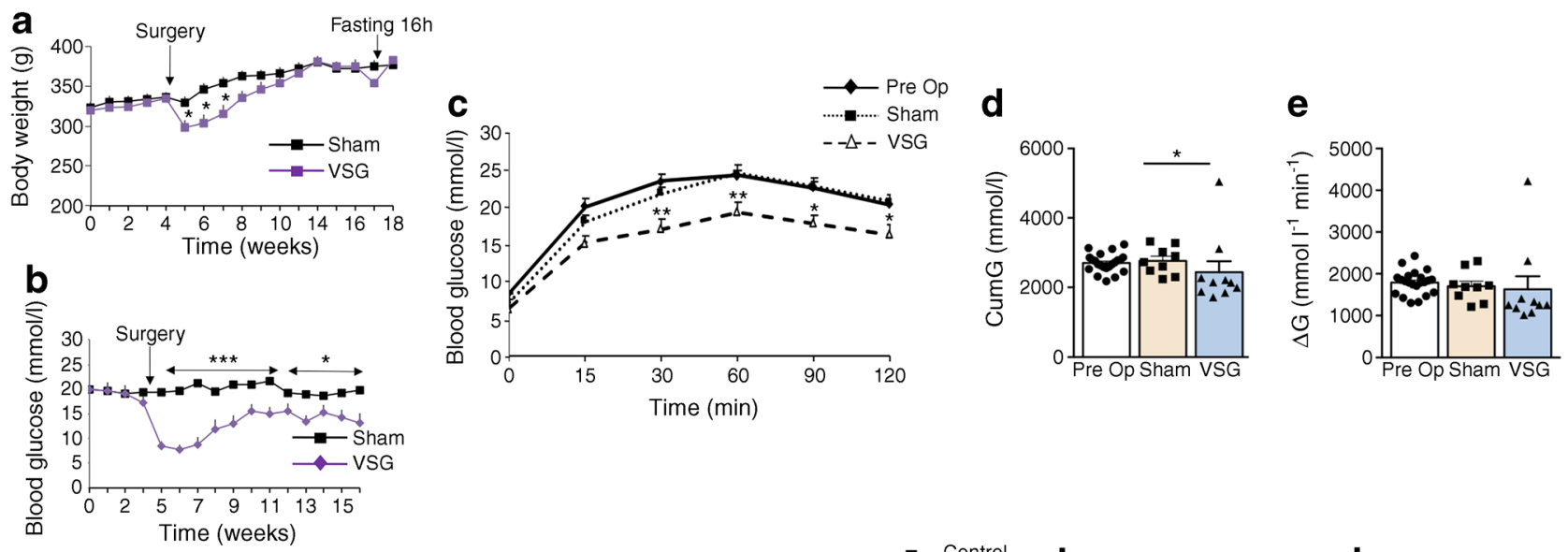
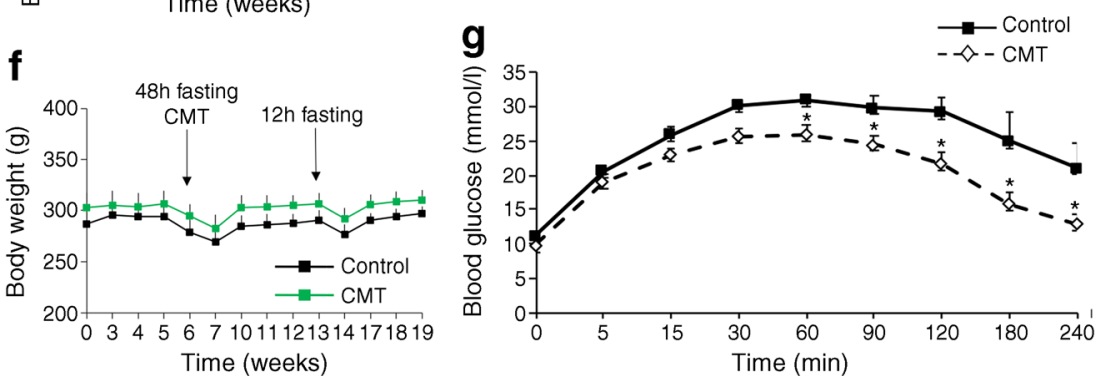

h
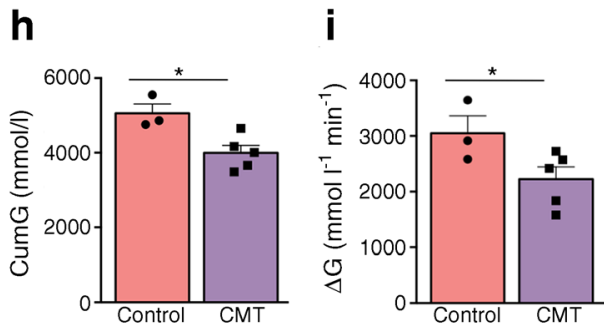

Fig. 2 Effects of VSG and gut microbiota transfer in gastrectomised GK rats. Changes in body weight (a, f), blood glucose (b) and glucose tolerance $(\mathbf{c}-\mathbf{e} ; \mathbf{g}-\mathbf{i})$ in GK rats following VSG or sham operation $(\mathbf{a}-\mathbf{e})$, and following inoculation of GK rats with gut microbiota from gastrectomised GK rats or sham controls $(\mathbf{f}-\mathbf{i})$. OGTTs $(\mathbf{c})$ were performed following an overnight $(16 \mathrm{~h}$ ) fast, before VSG (Pre Op) and 91 days after VSG $(n=10)$ or sham operation $(n=9)(\mathbf{c}-\mathbf{e})$. IPGTTs $(\mathbf{g})$ were performed 12 days after caecal microbiota transfer (CMT) from
VSG-treated GK rats $(n=5)$ or sham-operated GK rats (controls; $n=3$ 4) $(\mathbf{g}-\mathbf{i})$. Data are mean \pm SEM. The Kruskal-Wallis test was used to analyse glucose tolerance data. The non-parametric Mann-Whitney $U$ test was used to analyse the other variables. $* p<0.05, * * p<0.01$, $* * * p<0.001$ vs sham-operated GK rats $(\mathbf{a}-\mathbf{e})$ or GK rats inoculated with microbiota from sham-operated GK rats $(\mathbf{g}-\mathbf{i})$. CumG, cumulative glucose levels during the glucose tolerance test; $\Delta \mathrm{G}$, cumulative glucose levels above baseline 


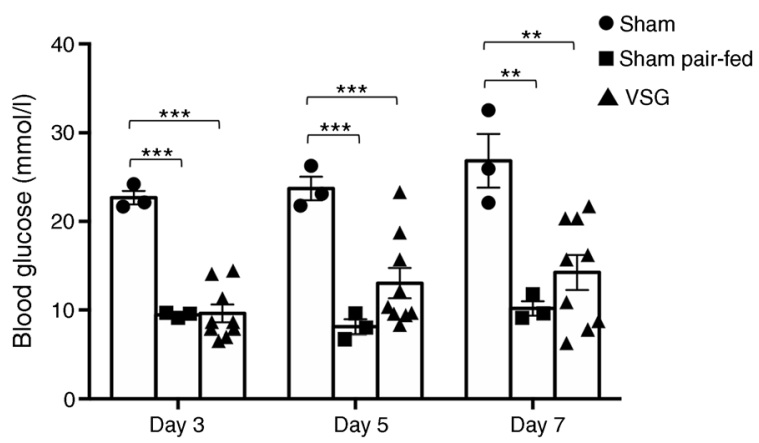

Fig. 3 Impact of caloric restriction on blood glucose in sham-operated GK rats. Blood glucose was determined ad libitum in a group of shamoperated GK rats $(n=3) 3,5$ and 7 days after the beginning of the pairfeeding experiment and compared with free-fed sham-operated $(n=3)$ and gastrectomised (VSG; $n=9$ ) GK rats. Non-parametric MannWhitney $U$ tests were applied for statistical data analysis. ${ }^{*} p<0.01$, $* * * p<0.001$ vs sham-operated GK rats

VSG and dietary restriction are equally efficient to reduce hyperglycaemia in GK rats To evaluate the performance of VSG in improving glucose homeostasis in the GK strain, we carried out a pair-feeding experiment in GK rats 30 days after the sham operation. Blood glucose was systematically significantly reduced in caloric restricted sham-operated GK rats when compared with free-fed sham-operated GK rats (Fig. 3). Interestingly, the magnitude of the drop in glucose levels following caloric restriction in sham-operated GK rats and VSG in GK rats was very similar, demonstrating the efficiency of VSG in improving glucose homeostasis.

VSG alters gut microbiota architecture To identify changes in the gut microbiota architecture that may explain VSG-induced improvement in glucose homeostasis, we carried out metagenome sequencing in gastrectomised and shamoperated GK rats. We sequenced bacterial DNA from caecum and colon content collected 14 weeks after VSG when glucose intolerance was reduced in gastrectomised GK rats, whilst body weight returned to control values (Table 1). These intestinal sites are the richest in bacteria and most active for nutrient absorption. The 27 microbiota samples generated a consistent amount of sequencing data ranging from $21.6 \mathrm{~Gb}$ per sample in the colon of VSG-treated rats to $22.9 \mathrm{~Gb}$ in the caecum of control rats (Table 1). Considering the high technical reproducibility of each sample across the four sequencing runs (ESM Fig. 3) data from technical replicates were pooled.

The depth of sequencing allowed for over 16,000 observations of rDNA motifs per caecum and colon sample (Table 1) thus providing confidence for detecting any bacterial species present at 1:5000 or better. Over 1200 unique rDNA motifs were identified in each sample (range 1281-1427) (Table 1). Overall, sequencing data in all samples and all intestinal sites identified 8767 unique rDNA motifs (517,697 occurrences).

We used these data to assess sequencing data consistency in the caecum and colon within individual animals and subsequently caecum-colon, caecum-caecum and colon-colon between animals of the same group. Mean and SD of the 100 most common 16S rDNA sequences illustrate the relatively large variability of data from the most frequent motifs in caecum and colon (ESM Figs $4 \mathrm{a}, \mathrm{b}$ and $5 \mathrm{a}, \mathrm{b}$ ). Data from caecum and colon from the same animal were generally more similar (up to 99\%) than any combination from different animals (ESM Figs $4 \mathrm{c}$ and $5 \mathrm{c}$ ). For samples with paired caecum-colon microbiome sequencing datasets, comparisons of the 10 most prevalent rDNA motifs across all samples illustrate that within-individual similarity is higher than betweenindividual similarity (ESM Fig. 6).

We carried out exact match searches for a $55 \mathrm{mer}$ sequence beginning with the sequence of the V13A reverse primer and selected 232 rDNA motifs present at an occurrence of $>0.005 \%$ in at least $50 \%$ of individuals, regardless of the sample's intestinal origin (ESM Table 2). Despite the large
Table 1 Details of metagenome sequencing of caecum and colon samples from gastrectomised and sham-operated GK rats

\begin{tabular}{llllll}
\hline & \multicolumn{2}{l}{ VSG } & & \multicolumn{2}{l}{ Sham } \\
\cline { 2 - 3 } \cline { 5 - 6 } Intestinal site & Caecum (10) & Colon (6) & & Caecum (8) & Colon (3) \\
\hline Mean length $(\mathrm{Gb})$ & $22.4 \pm 3.7$ & $21.6 \pm 3.6$ & & $22.9 \pm 3.1$ & $21.8 \pm 1.1$ \\
Total rDNA motifs & $16,253 \pm 3940$ & $18,485 \pm 6478$ & & $16,138 \pm 3671$ & $16,067 \pm 1089$ \\
Unique rDNA motifs & $1302 \pm 156$ & $1281 \pm 170$ & & $1367 \pm 167$ & $1427 \pm 89$ \\
Frequency $>5 \%$ & $1.25(38.38)$ & $0.31(28.13)$ & & $1.25(27.98)$ & $0.32(8.00)$ \\
Frequency $0.5-5 \%$ & $8.10(35.54)$ & $9.97(52.57)$ & & $10.28(39.18)$ & $11.21(55.49)$ \\
Frequency $<0.5 \%$ & $90.65(23.01)$ & $89.72(18.78)$ & & $88.47(23.98)$ & $88.47(19.29)$ \\
\hline
\end{tabular}

Data are mean \pm SD or frequency (proportion)

The number of biological replicates for each treatment group and intestinal site is given in parentheses

The total amount of sequencing data obtained (mean length, in $\mathrm{Gb}$ ) is given. Similar numbers of total and unique rDNA motifs were found in the four groups

The proportion of highly frequent $(>5 \%)$, moderately frequent $(0.5-5 \%)$ and rare $(<0.5 \%)$ motifs was calculated. The proportion of the gut microbiome that these categories account for is given in parentheses 
numbers of unique rDNA motifs identified in the dataset, the 52 most prevalent rDNA motifs accounted for $80 \%$ of the total data. Given the depth of metagenome sequencing and extensive within-individual similarities in rDNA motifs in caecum and colon, caecal sequence data were used in analyses of $16 \mathrm{~S}$ rDNA motif enrichment. Each individual microbiome was dominated by few rDNA present at a frequency $>5 \%$ in gastrectomised and control rats (Fig. 4a, b, ESM Table 2, ESM Fig. 7), representing only $0.31-1.25 \%$ of the motifs, but accounting for a large proportion of the overall motif abundance (up to $38.38 \%$ in the caecum of gastrectomised rats) (Table 1). In contrast, the largest diversity of the microbiome, representing over $88 \%$ of distinct motifs, was accounted for by motifs present at very low frequency $(<0.5 \%)$ and collectively covering only $18.78-23.98 \%$ of the sequenced microbiome (Table 1).
$P$. copri dominates GK gut microbiota and is enriched by VSG We next searched for differentially enriched rDNA motifs in gastrectomised and control rats. We identified ten motifs overrepresented in VSG-treated rats and 16 motifs overrepresented in controls (Table 2). Two motifs were present only in the gut microbiome of sham (V13A9832) or VSG (V13A7019) rats. The most frequent rDNA motif (V13A7759), accounting for up to $54.5 \%$ of the microbiome of individual samples, was significantly over-represented in gastrectomised GK rats $(22.71 \%)$ than in controls $(9.07 \%$, $p=0.046$ ) (Table 2, Fig. 4b). Sequence homology searches in microbial genome databases identified only 9 rDNA motifs showing maximum sequence similarities (100\%) with the DNA sequence of a single (V13A7759, V13A9569, V13A12536, V13A6709, V13A399, V13A234, V13A622) or several (V13A1861, V13A12857) bacteria (Table 2). The

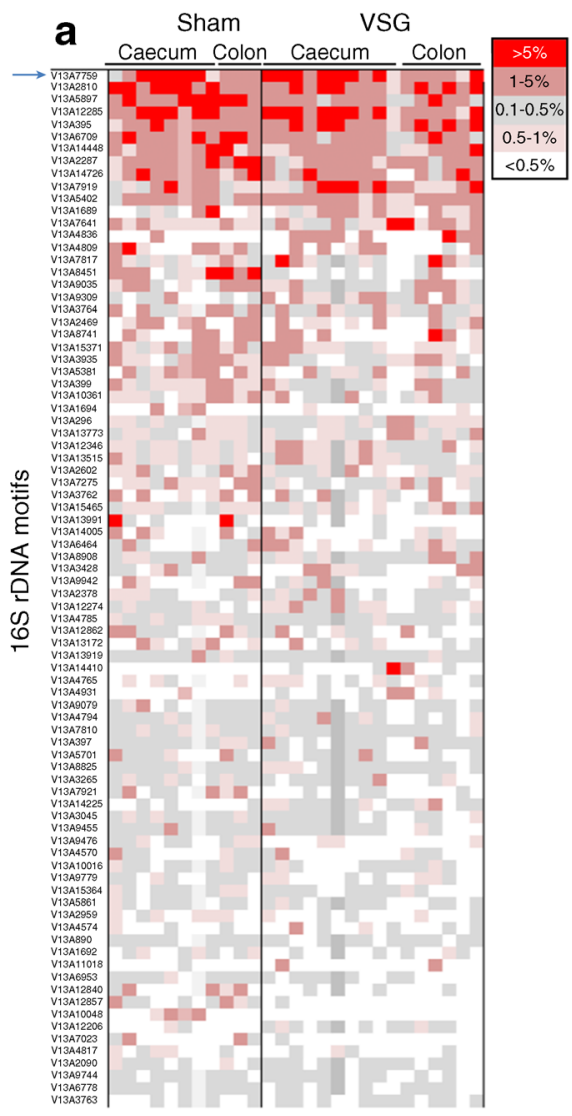

b

Caecum
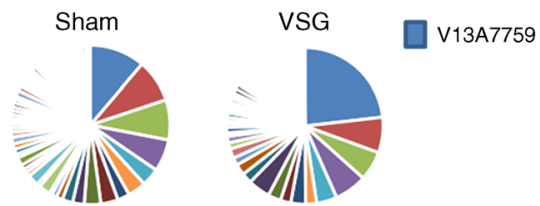

Total
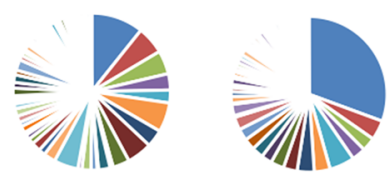

Colon

C
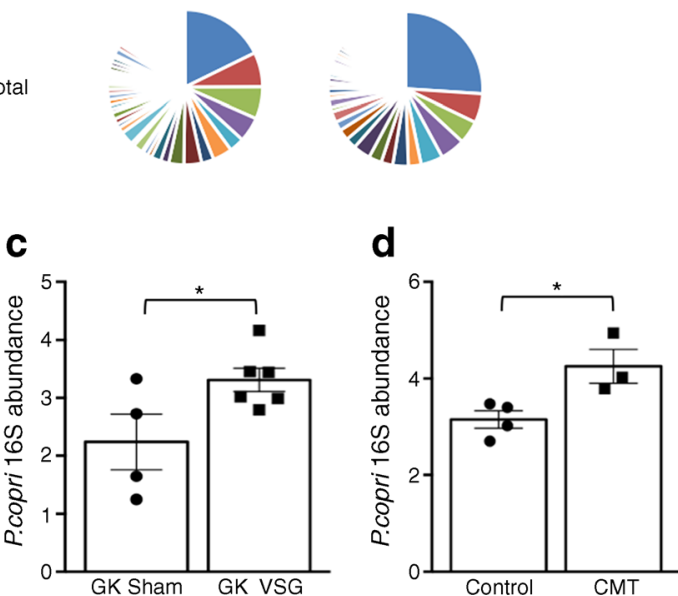

Fig. 4 Effects of VSG on gut microbiome architecture in the GK rat. Frequency of $16 \mathrm{~S}$ rDNA motifs derived from metagenome sequencing was calculated in each caecum and colon sample from GK rats following VSG $(n=10)$ or sham operation $(n=8)(\mathbf{a})$. Means of rDNA motif frequencies were calculated for data from caecum, colon and caecum and colon combined in the two rat groups (b). Data are shown for the 84 most abundant motifs. Each line in (a) represents a different motif colour-coded according to its frequency and each colour in (b) represents the relative proportion of each motif; the arrow in (a) and the blue colour in (b) represent V13A7759. Quantitative RT-PCR was carried out to

assess enrichment of the motif V13A7759 (P. copri) in GK rats following VSG $(n=6)(\mathbf{c})$ and GK rats following caecal microbiota transfer (CMT) from VSG-treated GK rats $(n=3)(\mathbf{d}) ; n=4$ for sham/control in both experiments. Details of all rDNA motifs, associated frequencies and statistical differences between the rat groups are given in ESM Table 2. Data are mean \pm SEM. Non-parametric Mann-Whitney $U$ tests were applied for statistical analysis of $P$. copri abundance. $* p<0.05$ vs shamoperated GK rats (c) or GK rats inoculated with microbiota from shamoperated GK rats $(\mathbf{d})$ 
Table 2 Frequency of rDNA motifs significantly enriched in the gut microbiota from gastrectomised (A) or sham-operated (B) GK rats

\begin{tabular}{|c|c|c|c|c|c|c|}
\hline & Motif & Sham & VSG & $p$ value & Sequence homology with bacteria & Accession \\
\hline \multirow[t]{14}{*}{$\mathrm{A}(\%)$} & V13A7759 & $9.074 \pm 3.114$ & $22.706 \pm 5.539$ & 0.046 & Prevotella copri DSM 18205 & NZ_GG703862 \\
\hline & V13A9309 & $0.350 \pm 0.243$ & $1.017 \pm 0.228$ & 0.015 & - & - \\
\hline & V13A14410 & $0.003 \pm 0.002$ & $0.636 \pm 0.548$ & 0.002 & - & - \\
\hline & V13A14225 & $0.064 \pm 0.047$ & $0.310 \pm 0.097$ & 0.020 & - & - \\
\hline & V13A12206 & $0.142 \pm 0.116$ & $0.206 \pm 0.053$ & 0.036 & - & - \\
\hline & V13A1861 & $0.031 \pm 0.008$ & $0.124 \pm 0.024$ & 0.002 & Mannheimia haemolytica M42548 & NC_021082 \\
\hline & & & & & Mannheimia succiniciproducens MBEL55E & NC_006300 \\
\hline & & & & & Necropsobacter massiliensis strain FF6 & NZ_CDON01000005 \\
\hline & & & & & Pasteurella bettyae CCUG 2042 & NZ_AJSX01000044 \\
\hline & & & & & Rodentibacter heylii strain $1,998,236,014$ & $\mathrm{NZ}_{\overline{-}}{ }_{\mathrm{M} A A 01000050}$ \\
\hline & V13A11382 & $0.010 \pm 0.010$ & $0.088 \pm 0.039$ & 0.014 & - & - \\
\hline & V13A9569 & $0.012 \pm 0.003$ & $0.035 \pm 0.007$ & 0.008 & Anaerotignum lactatifermentans DSM 14214 & NZ_FRAH01000002 \\
\hline & V13A12536 & $0.001 \pm 0.001$ & $0.020 \pm 0.008$ & 0.018 & Planococcus halocryophilus Or1 & NZ_CP016537 \\
\hline & V13A7019 & Absent & $0.008 \pm 0.004$ & 0.020 & - & - \\
\hline \multirow[t]{19}{*}{$\mathrm{B}(\%)$} & V13A6709 & $3.043 \pm 0.538$ & $1.494 \pm 0.237$ & 0.046 & Prevotella pleuritidis JCM 14110 & NZ_BAJN01000030 \\
\hline & V13A8451 & $1.814 \pm 0.615$ & $0.096 \pm 0.040$ & 0.008 & - & - \\
\hline & V13A399 & $0.902 \pm 0.117$ & $0.484 \pm 0.167$ & 0.011 & Planktothrix agardhii NIVA-CYA 126/8 & NZ_CM002803 \\
\hline & V13A10048 & $0.665 \pm 0.266$ & $0.004 \pm 0.003$ & 0.033 & - & - \\
\hline & V13A12857 & $0.286 \pm 0.141$ & $0.023 \pm 0.006$ & 0.014 & Eubacterium eligens ATCC 27750 & NC_012778 \\
\hline & & & & & Eubacterium hallii DSM 3353 & NZ_ACEP01000116 \\
\hline & & & & & Eubacterium ramulus ATCC 29099 & NZ_KI271105 \\
\hline & & & & & Lactobacillus rogosae strain ATCC 27753 & NZ_FONU01000001 \\
\hline & V13A9832 & $0.273 \pm 0.097$ & Absent & 0.021 & - & - \\
\hline & V13A234 & $0.148 \pm 0.031$ & $0.071 \pm 0.058$ & 0.019 & Bacteroides paurosaccharolyticus JCM 15092 & NZ_BAJR01000054 \\
\hline & V13A686 & $0.137 \pm 0.052$ & $0.022 \pm 0.011$ & 0.036 & - & - \\
\hline & V13A603 & $0.136 \pm 0.057$ & $0.018 \pm 0.018$ & 0.037 & - & - \\
\hline & V13A7033 & $0.130 \pm 0.019$ & $0.065 \pm 0.009$ & 0.011 & - & - \\
\hline & V13A622 & $0.075 \pm 0.036$ & $0.015 \pm 0.005$ & 0.048 & Ruminococcus flavefaciens MC2020 & NZ_JNKE01000007 \\
\hline & V13A1295 & $0.073 \pm 0.015$ & $0.035 \pm 0.011$ & 0.046 & - & - \\
\hline & V13A6720 & $0.032 \pm 0.013$ & $0.005 \pm 0.003$ & 0.019 & - & - \\
\hline & V13A1442 & $0.024 \pm 0.006$ & $0.001 \pm 0.001$ & 0.002 & - & - \\
\hline & V13A6490 & $0.018 \pm 0.007$ & $0.004 \pm 0.002$ & 0.012 & - & - \\
\hline & V13A12243 & $0.011 \pm 0.004$ & $0.002 \pm 0.001$ & 0.025 & - & - \\
\hline
\end{tabular}

Data are shown as means of motif frequency \pm SEM

Sequences of the rDNA motifs are given in ESM Table 2, along with data from the set of rDNA motifs present in caecum samples in at least $50 \%$ of individual rats at a frequency $>0.005 \%$. Only known bacteria showing full sequence homology with rDNA motifs are given

most predominant motif in GK rats (V13A7759) is homologous to scaffold assemblies of $P$. copri (Table 2). Its significant enrichment in gastrectomised GK rats and after inoculation of microbiota from gastrectomised GK rats was confirmed by quantitative PCR of caecum DNA (Fig. 4c, d).

Intestinal $P$. copri enrichment improves glucose regulation in GK rats Owing to the controversial role of $P$. copri on cardiometabolic phenotypes [21, 22], we focused functional studies on this bacterial species, which was one of the few commercially available species differentially represented in our model systems. To test the hypothesis of a role of $P$. copri on improved glucose regulation, we treated GK rats with antibiotics (vancomycin and kanamycin) permitting its growth and resulting in $P$. copri intestinal enrichment [23] (Experiment 3 in Fig. 1).We verified that antibiotics significantly reduced bacterial DNA concentration in faeces (Fig. 5a), thus inhibiting the gut bacterial ecosystem, and that it significantly increased the proportion of $P$. copri (Fig. 5b). Antibiotics did not affect body weight (ESM Fig. 8a), but significantly 

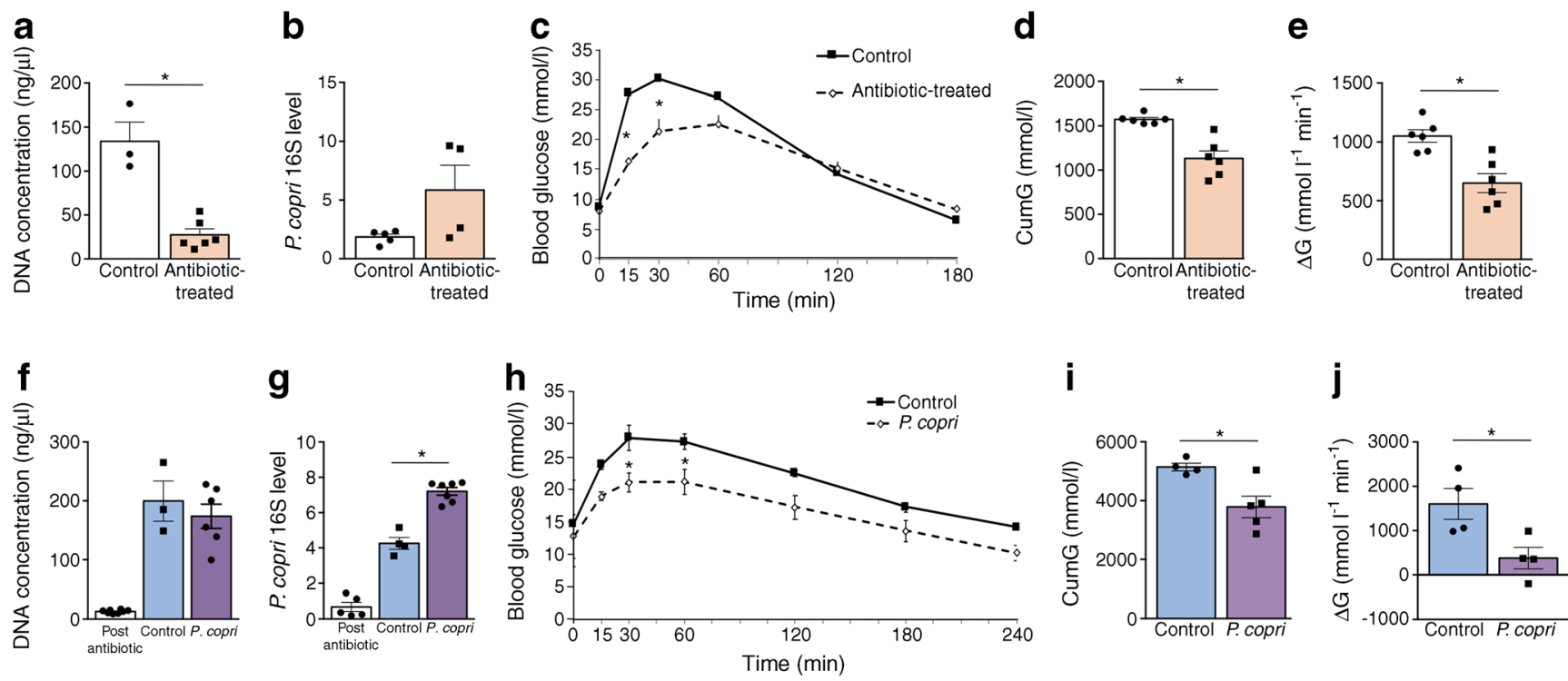

Fig. 5 Effects of gut microbiota enrichment in P. copri on glucose homeostasis in GK rats. GK rats were either treated with antibiotics permissive to $P$. copri or remained in antibiotic-free conditions (a-e). A separate group of GK rats was inoculated with $P$. copri or heat-inactivated P. copri $(\mathbf{f}-\mathbf{j})$. Faecal DNA concentration and P. copri abundance were determined in antibiotic-treated $(n=3-6)$ and control $(n=4-6)$ rats $(\mathbf{a}, \mathbf{b})$. Faecal DNA concentration and P. copri abundance were determined in GK rats treated with a combination of broad spectrum antibiotics prior to inoculation (post antibiotics) $(n=5)$, and in rats inoculated with $P$. copri
( $n=6)$ or heat-inactivated P. copri (control) $(n=3-4)(\mathbf{f}, \mathbf{g})$. Glucose tolerance $(\mathbf{c}-\mathbf{e}, \mathbf{h}-\mathbf{j})$ was determined following an IPGTT in overnight fasted $(16 \mathrm{~h}) \mathrm{GK}$ rats 10 days after antibiotic treatment or $P$. copri inoculation. Data are mean \pm SEM. The Kruskal-Wallis test was used to analyse glucose tolerance data. The non-parametric Mann-Whitney $U$ test was used to analyse the other variables. ${ }^{*} p<0.05$ vs control. CumG, cumulative glucose levels during the IPGTT; $\Delta \mathrm{G}$, cumulative glucose levels above baseline improved glucose tolerance. Antibiotic treatment resulted in significant reduction in blood glucose 15 and $30 \mathrm{~min}$ after intraperitoneal glucose injection (Fig. 5c), in cumulative blood glucose (Fig. 5d) and in the $\Delta$ G parameter (Fig. 5e) when compared with untreated GK controls. Glucose-induced insulin secretion was similar in the two rat groups (ESM Fig. 9a).

\section{$P$. copri inoculation improves glucose regulation in GK rats To} further assess the impact of $P$. copri enrichment on glucose homeostasis, we inoculated GK rats with $5 \times 10^{8} \mathrm{CFU}$ P. copri or with heat-killed P. copri (Experiment 4 in Fig. 1). We initially measured faecal DNA concentration to verify microbiota abolition by the antibiotics. We then verified by PCR that gut microbiota enrichment in $P$. copri was implemented 12 days after bacterial inoculation (Fig. 5f, g). P. copri treatment had no effect on body weight (ESM Fig. 8b), but was associated with a significant reduction of glucose intolerance when compared with GK rats treated with heat-inactivated P. copri. Blood glucose after intraperitoneal glucose injection was lower in P. copri-treated GK rats than in controls (Fig. $5 \mathrm{~h}) . P$. copri inoculation resulted in a significant reduction in cumulative blood glucose levels during the glucose tolerance test (Fig. $5 \mathrm{i}$ ) and in the $\Delta \mathrm{G}$ parameter (Fig. 5j). P. copri had no significant impact on insulin secretion (ESM Fig. 9b).

Our data demonstrate that increased representation of intestinal $P$. copri in GK rats improves glucose homeostasis.
A gut microbiota enriched in $P$. copri stimulates bile acid metabolism Given the consistent effects of bariatric surgery on improved glucose tolerance and increased circulating bile acids in humans [24], we hypothesised that $P$. copri intestinal enrichment in GK rats stimulates bile acid metabolism. Mass spectrometry allowed the quantification of $27-35$ bile acids in plasma from all rat groups, which were analysed as individual molecules (ESM Tables 3 and 4) and total, primary and secondary bile acids (Fig. 6). VSG in GK rats significantly increased concentrations of ursocholanic, tauro-ursodeoxycholic ( $5 \beta$-cholanic acid- $3 \alpha, 7 \beta$ diol $N$-(2-sulphoethyl)) and taurohyodeoxycholic (5ß-cholanic acid-3 $\alpha, 6 \alpha$-diol $N$-(2-sulphoethyl)-amide) acids (ESM Table 3). Total and primary bile acid concentrations were similar in gastrectomised and sham-operated rats (Fig. 6a, b). The level of secondary bile acids, particularly deoxycholic acid, showed a 2.8 fold increase in VSG rats leading to a significant decrease in the ratio primary to secondary bile acids in gastrectomised rats $(p=$ 0.030).

Likewise, caecal microbiota transfer from gastrectomised GK to GK rats was associated with significantly increased concentrations of ursocholanic, tauro-ursocholanic (5ß-cholanic acid $\mathrm{N}$-(2sulphoethyl)-amide)) and isolithocholic acids (ESM Table 4). Treated GK rats showed a marked stimulation of total $(+239 \%)$, primary $(+214 \%)$ and secondary (+260\%) bile acids (Fig. 6e-g). In contrast, many bile acids were downregulated by $P$. copri permissive antibiotics in GK rats, resulting in the downregulation 
Fig. 6 Effects of intestinal P. copri enrichment on plasma bile acid concentrations in the GK rat. Mass spectrometry methods were used to determine the plasma concentration of bile acids in GK rats following VSG $(n=8)$ or sham operation $(n=5)(\mathbf{a}-\mathbf{d})$ in GK rats following caecal microbiota transfer (CMT) from VSG-treated GK rats $(n=5)$ or sham-operated GK rats $(n=4)(\mathbf{e}$ h), in GK rats treated with a combination of kanamycin and vancomycin antibiotics $(n=5)$ or in the absence of antibiotics in controls $(n=4)(\mathbf{i}-\mathbf{I})$, or in GK rats inoculated with $P$. copri $(n=6)$ or heat-inactivated $P$. copri $(n=3)$ (m-p). Non-parametric MannWhitney $U$ tests were used for statistical analysis. Data are mean \pm SEM. $* p<0.05, * * p<0.01$, ${ }^{\dagger} p=0.05,{ }^{\star} p=0.06,{ }^{\circledR} p=0.07$ vs the relevant control. BA, bile acid

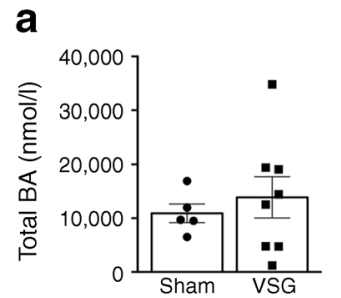

e

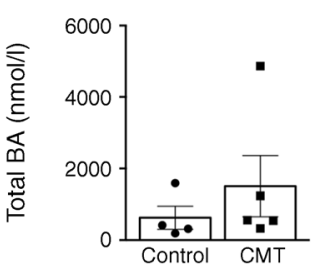

i

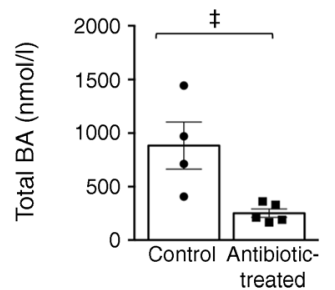

m

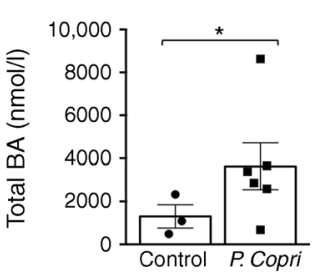

b

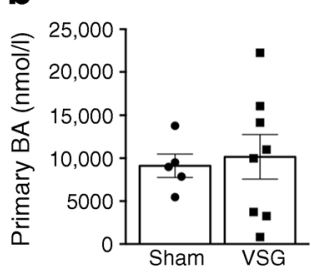

f

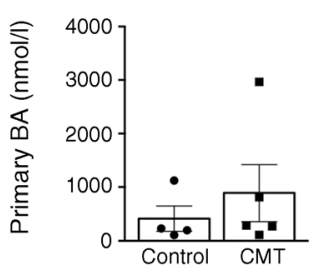

j

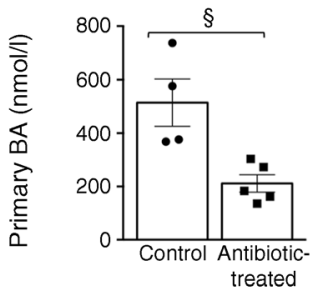

n

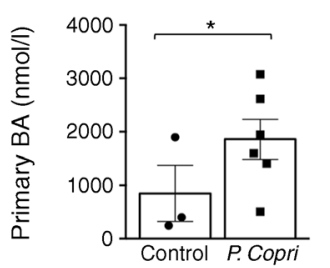

C

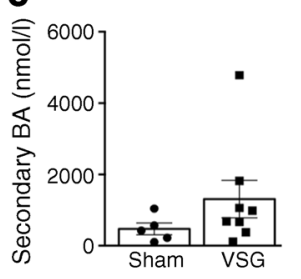

g

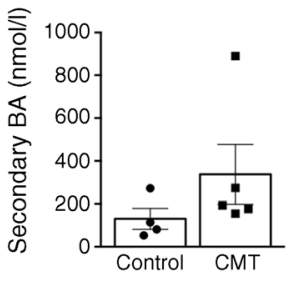

k

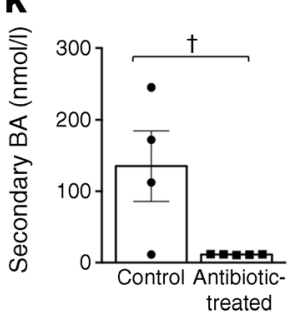

0

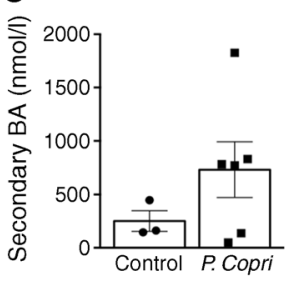

d

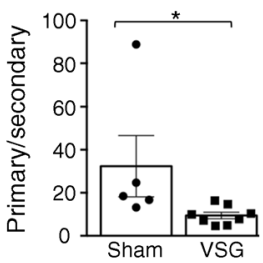

h

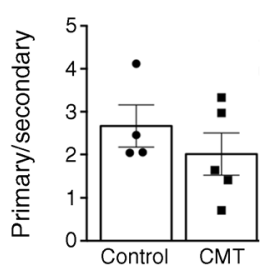

I

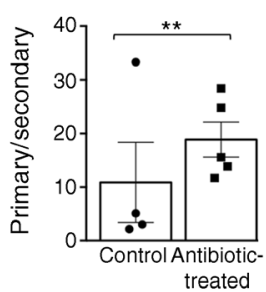

p

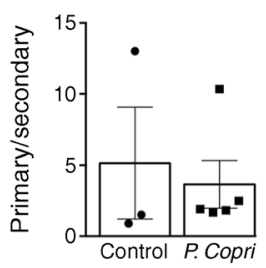

of total, primary and secondary bile acids, and a 5.5-fold increase in the ratio of primary to secondary bile acids $(p=0.007$ ) (ESM Table 4) (Fig. 6i-k). Finally, P. copri inoculation in GK rats increased the concentrations of many bile acids, including cholic $(+518 \%, p=0.008)$, allolithocholic $(+454 \%, p=0.016)$, chenodeoxycholic $(+341 \%, p=0.048)$ and $\omega$-muricholic $(+810 \%, p=0.027)$ acids, resulting in significantly increased total $(+460 \%, p=0.047)$ and primary $(+492 \%, p=0.010)$ bile acids (ESM Table 4; Fig. 6m, n).

Overall, bile acids were stimulated upon intestinal enrichment of $P$. copri. Interestingly $P$. copri inoculation was associated with increased levels of primary bile acids chenodeoxycholic acid $(+341 \%, p=0.048)$ and cholic acid $(+610 \%, p=0.009)(\mathrm{ESM}$ Table 4), which activate FXR [24, 25].

Enhanced liver expression of FXR is associated with stimulation of bile acid metabolism and improved glucose tolerance consecutive to intestinal $P$. copri enrichment FXR activation has been consistently reported in response to bariatric surgery and associated stimulation of circulating bile acids [26]. We analysed the expression of Fxr (also known as NrIh4) and genes relevant to its function in liver and adipose tissue of the four models generated in our study. Liver expression of $F x r$ was significantly upregulated in gastrectomised GK rats and in GK rats inoculated with gut microbiota from VSG GK rats or with $P$. copri, when compared with corresponding controls (Fig. 7a-d). A primary target of FXR (Shp [also known as NrOb2]) and G6pc, which encodes glucose 6 phosphatase exhibited direction of expression changes similar to those of Fxr in the four rat groups. Expression of Pepck (encoding phosphoenolpyruvate carboxykinase) and $C y p 7 a 1$ (encoding cholesterol $7 \alpha$-hydroxylase [CYP7A1]/cytochrome P450), which are normally downregulated by bile acids and FXR, were inhibited in gastrectomised GK rats (Pepck and Cyp7al), in GK rats treated with the gut flora of VSG GK rats (Pepck) and in GK rats inoculated with P. copri (Cyp7a1). Expression of the transcription factors Chrebp (also known as Mlxipl) and Srebf1, which regulate liver lipogenesis and cholesterol biogenesis, was significantly stimulated in VSG rats, in GK rats treated with $P$. copri permissive 

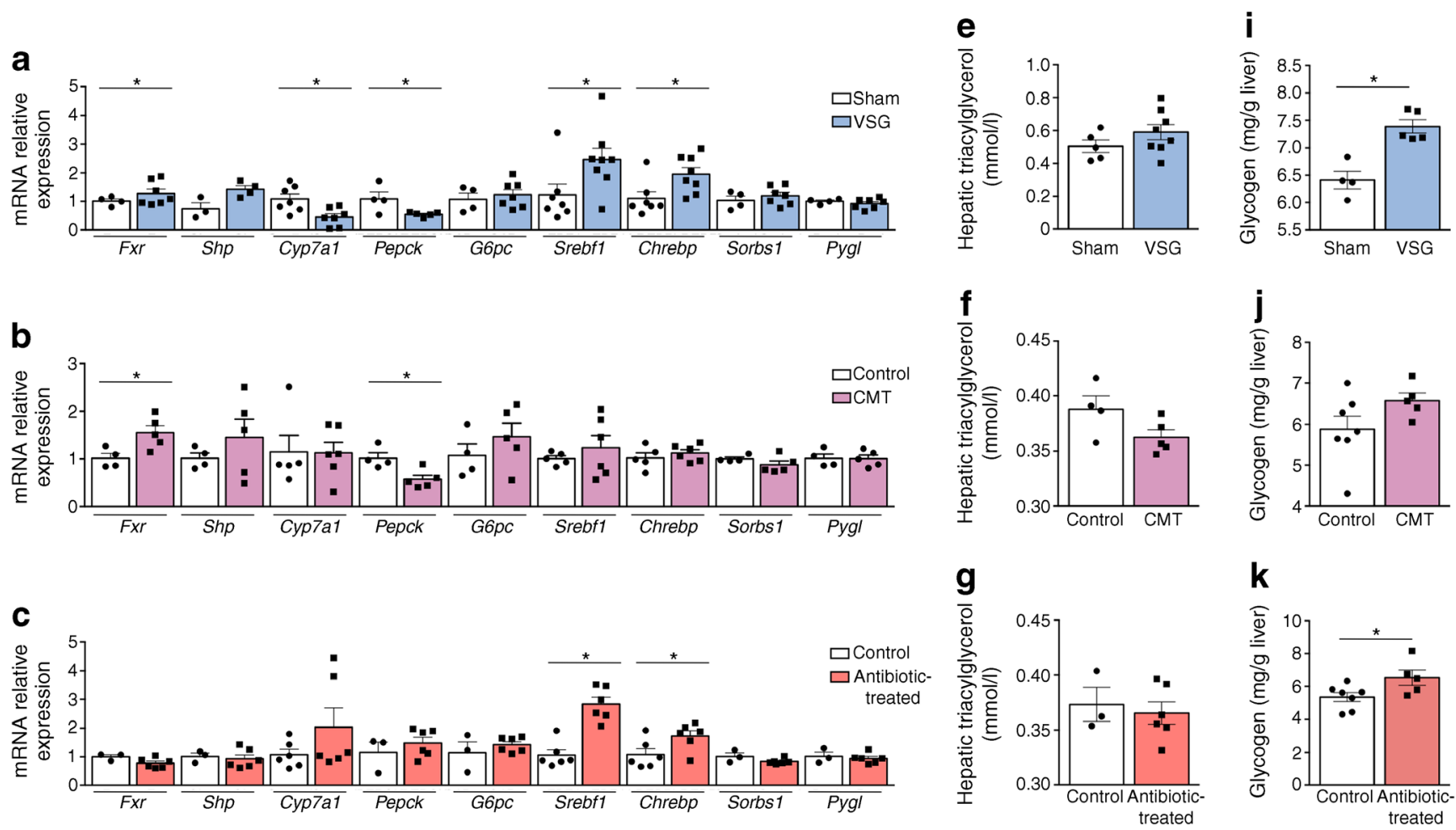

d
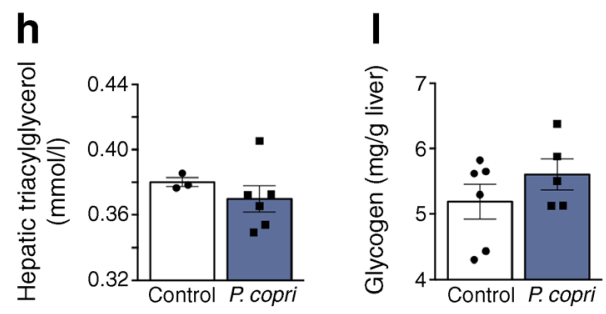

Fig. 7 Liver gene expression and hepatic triacylglycerol and glycogen storage in treated GK rats and controls. Liver gene expression (a-d) assessed by quantitative RT-PCR and quantification of hepatic triacylglycerol content $(\mathbf{e}-\mathbf{h})$ and glycogen storage $(\mathbf{i}-\mathbf{l})$ were determined in GK rats following VSG (a, e, i), in GK rats following caecal microbiota transfer (CMT) from VSG-treated GK rats $(\mathbf{b}, \mathbf{f}, \mathbf{j})$, in GK rats following treatment with $P$. copri permissive antibiotics $(\mathbf{c}, \mathbf{g}, \mathbf{k})$ and in GK rats following inoculation with $P$. copri $(\mathbf{d}, \mathbf{h}, \mathbf{I}), n=4-10$ per group. Data are mean \pm SEM. Non-parametric Mann-Whitney $U$ tests were used for statistical analysis. ${ }^{*} p<0.05$ vs sham/control rats antibiotics and in GK rats inoculated with P. copri (Fig. 7a, c, d). Expression of Sorbs 1, which is involved in insulin signalling, and $P y g l$, which encodes glycogen phosphorylase, was unaffected by VSG and by increased intestinal $P$. copri levels.

To test the functional relevance of stimulated $F x r$ transcription in the rat models, we analysed the hepatic concentration of triacylglycerol (Fig. 7e-h) and glycogen (Fig. 7i-l), which are both regulated by FXR. Triacylglycerol levels were unchanged in the experimental groups (Fig. 7e-h). In contrast, hepatic glycogen content was significantly more elevated in gastrectomised GK rats (Fig. 7i) and in GK rats treated with the $P$. copri permissive antibiotics (Fig. $7 \mathrm{k}$ ) than in corresponding controls.

Finally, considering the functional consequences of bariatric surgery and FXR activation on adipose tissue metabolism and inflammation [27, 28], we analysed the expression of relevant genes in the adipose tissue in GK rats in response to VSG and enhanced intestinal P. copri (ESM Fig. 10). Consistent with the liver data, Fxr and Srebf1 expression was co-ordinately upregulated in adipose tissue following VSG (ESM Fig. 10a). Treatment with $P$. copri permissive antibiotics and inoculation of $P$. copri in GK rats also resulted in stimulation of $F x r$ and Srebfl expression (ESM Fig. 10c, d). Expression of Tnfa was unchanged in all four experimental groups. In contrast, downregulated expression of $I l 6$ in GK rats inoculated with the microbiota of gastrectomised GK rats (ESM Fig. 10b) or with P. copri (ESM Fig. 10d) and upregulated expression of $I l 10$ in all four rat models (ESM Fig. 10a-d), suggest antiinflammatory effects of $P$. copri.

Collectively, these data underline the role of intestinal $P$. copri in transcriptional regulation of FXR signalling, which contributes to improve glucose and lipid homeostasis. 


\section{Discussion}

Our data underline the long term effects of VSG on gut microbiota architecture remodelling and the reduction of glucose intolerance in the non-obese GK rat model of polygenic type 2 diabetes, which therefore avoids the confounding effects of weight loss induced by gastrectomy in individuals with both diabetes and obesity. Our data suggest that VSG may be equivalent, or even superior, to caloric restriction, which often results in only transient improvement in glucose homeostasis in diabetic patients [29]. Our results contribute to our understanding of the relationship between gastrectomy and improved glucose homeostasis, by demonstrating the involvement of a specific bacterial species (P. copri), which is stimulated by VSG, in the regulation of glucose tolerance and the activation of FXR and bile acid metabolism in the GK strain.

A causal relationship between cardiometabolic diseases and gastrointestinal microbiota has been inferred through associations between reduced disease risk and increased bacteria diversity and gene richness [30,31] and the identification of bacteria associated with surgically-induced remission of diabetes in obese patients [32] and in preclinical models [33-36]. The most abundant rDNA motif enriched in gastrectomised GK rats aligned to the draft genome assembly of $P$. copri. Considering the dominance of $P$. copri in both GK and gastrectomised GK rats, gene richness is unlikely to significantly account for improved glucose homeostasis following VSG. On the other hand, our findings in GK rats carrying enriched intestinal $P$. copri abundance support the causal relationship between intestinal P. copri and improved glucose metabolism.

Investigations into the pathophysiological role of $P$. copri have led to apparently contradictory results, which may be due to host diet-dependent effects and the existence of several P. copri clades [37]. Intervention studies have demonstrated that faecal levels of Prevotella correlate with high fibre and high carbohydrate dietary intake [38-40]. High fibre diet improves glucose homeostasis and increases faecal P. copri levels, resulting in increased liver glycogen in mice inoculated with human microbiota enriched in P. copri [22]. On the other hand, $P$. copri is correlated with insulin resistance in humans and in fat-fed mice [21]. Faecal P. copri is elevated in type 2 diabetes [41]. Improved glucose tolerance and increased circulating bile acids in GK rats following intestinal P. copri enrichment concur with its beneficial metabolic role. We hypothesise that naturally increased intestinal $P$. copri in the GK rat contributes to the complex aetiology of diabetes in this strain by counteracting the adverse effects of permanent hyperglycaemia, along with sequence variants in genes that stimulate insulin secretion [12] and downregulated expression of genes involved in heart failure [42].

Changes in plasma bile acid composition, combined with altered gut microbiota ecology, are hallmarks of the adaptation to bariatric surgery in humans and in preclinical models [32,
43]. Activation of FXR by bile acids stimulates the expression of the nuclear receptor SHP, which downregulates hepatic CYP7A1, the key enzyme in bile acid biosynthesis that converts cholesterol to $7 \alpha$-hydroxycholesterol $[26,44]$. Given the impact of bariatric surgery on bile acid metabolism and FXR signalling [45] and the association between increased P. copri abundance and glucose metabolism [22], we reasoned that improved glucose tolerance in response to increased intestinal $P$. copri abundance in GK rats involves FXR signalling. Upregulated expression of Fxr and Shp and downregulated expression of $C y p 7 a 1$, combined with increased bile acid synthesis and experimentally induced gut microbial enrichment in $P$. copri, supports our hypothesis.

Paralleled overexpression of Fxr and the transcription factors Chrebp and Srebplc (also known as Srebf1), which regulate the metabolism of triacylglycerols, fatty acids and phospholipids [46, 47], in both VSG-treated rats and GK rats inoculated with $P$. copri, may account for increased hepatic glycogen content identified in these models. Consistent overexpression of Fxr and Srebplc in adipose tissue of gastrectomised GK rats and in our models of $P$. copri enrichment was associated with systematic increase in $I l 10$ expression, suggesting activation of anti-inflammatory mechanisms.

Our findings warrant further investigation. Bacterial species present at low frequency in the GK gut, but significantly differentially enriched following VSG, may contribute to improve glucose homeostasis, either individually or as part of bacterial ecosystems. Conversely, antibiotic treatment increasing intestinal $P$. copri abundance may lead to coordinated changes in the abundance of bacterial species that can also regulate glucose tolerance, and remain to be identified. In addition, other molecular systems, including gut hormones, may also account for restored glucose homeostasis in gastrectomised GK rats. For example, Roux-en-Y gastric bypass (RYGB) in GK rats enhances insulin secretion and suppresses glucagon secretion, and restores islet structure through stimulation of the peptide tyrosine (PYY) [48].

Conclusions Our results illustrate interactions between the gut microbiome and host metabolism following surgical therapy for diabetes. We demonstrate that $P$. copri is the dominant intestinal bacterial species in the GK non-obese model of polygenic type 2 diabetes, which is further stimulated by gastrectomy, and that its intestinal enrichment mimics the effects of bariatric surgery on both elevated circulating bile acids and stimulated expression of FXR to reduce glucose intolerance. P. copri represents an attractive candidate for the development of probiotic-based therapies in diabetes. The exact genomic structure and gene content of $P$. copri remain to be established in order to elucidate the biological functions encoded by its genome that can account for its capacity to ferment dietary polysaccharides [49], its role in inflammation $[50,51]$ and its impact on host metabolism. 
Data availability All data generated or analysed during this study are included in this published article and its supplementary information files.

Funding This work was supported by grants from the European Commission (METACARDIS HEALTH-F4-2012-305312) and the Agence Nationale pour la Recherche (EpiTriO, ANR-15-EPIG-0002-05).

Authors' relationships and activities The authors declare that there are no relationships or activities that might bias, or be perceived to bias, their work.

Contribution statement EG, CM, ML, KD and DG conceived and designed the project. ALL, CR, FB, MV and CM performed animal procedures in GK rats, acquired physiological data and contributed to data analysis. NP, KD, FB and DG interpreted the results. KD, LH and JW produced and analysed metagenome sequencing data. AM and MED performed bile acid profiling. DG wrote the manuscript. NP, ALL and FB contributed equally to this work. All authors contributed to editing the article and approved the final version to be published. DG is responsible for the integrity of the work as a whole.

Open Access This article is licensed under a Creative Commons Attribution 4.0 International License, which permits use, sharing, adaptation, distribution and reproduction in any medium or format, as long as you give appropriate credit to the original author(s) and the source, provide a link to the Creative Commons licence, and indicate if changes were made. The images or other third party material in this article are included in the article's Creative Commons licence, unless indicated otherwise in a credit line to the material. If material is not included in the article's Creative Commons licence and your intended use is not permitted by statutory regulation or exceeds the permitted use, you will need to obtain permission directly from the copyright holder. To view a copy of this licence, visit http://creativecommons.org/licenses/by/4.0/

\section{References}

1. Sonnenburg JL, Bäckhed F (2016) Diet-microbiota interactions as moderators of human metabolism. Nature 535(7610):56-64. https://doi.org/10.1038/nature18846

2. Zmora N, Bashiardes S, Levy M, Elinav E (2017) The role of the immune system in metabolic health and disease. Cell Metab 25(3): 506-521. https://doi.org/10.1016/j.cmet.2017.02.006

3. Wu H, Tremaroli V, Bäckhed F (2015) Linking microbiota to human diseases: a systems biology perspective. Trends Endocrinol Metab 26(12):758-770. https://doi.org/10.1016/j.tem. 2015.09.011

4. Hansen TH, Gøbel RJ, Hansen T, Pedersen O (2015) The gut microbiome in cardio-metabolic health. Genome Med 7(1):33. https://doi.org/10.1186/s13073-015-0157-z

5. Brunkwall L, Orho-Melander M (2017) The gut microbiome as a target for prevention and treatment of hyperglycaemia in type 2 diabetes: from current human evidence to future possibilities. Diabetologia 60(6):943-951. https://doi.org/10.1007/s00125-0174278-3

6. Pollak M (2017) The effects of metformin on gut microbiota and the immune system as research frontiers. Diabetologia 60(9):1662 1667. https://doi.org/10.1007/s00125-017-4352-x

7. Mulla CM, Middelbeek RJW, Patti ME (2017) Mechanisms of weight loss and improved metabolism following bariatric surgery. Ann N Y Acad Sci 1411(1):53-64. https://doi.org/10.1111/nyas. 13409
8. Nguyen NT, Varela JE (2017) Bariatric surgery for obesity and metabolic disorders: state of the art. Nat Rev Gastroenterol Hepatol 14(3):160-169. https://doi.org/10.1038/nrgastro.2016.170

9. Schauer PR, Bhatt DL, Kirwan JP et al (2017) Bariatric surgery versus intensive medical therapy for diabetes - 5-year outcomes. N Eng1 J Med 376(7):641-651. https://doi.org/10.1056/ NEJMoa1600869

10. Goto Y, Kakizaki M, Masaki N (1976) Production of spontaneous diabetic rats by repetition of selective breeding. Tohoku J Exp Med 119(1):85-90. https://doi.org/10.1620/tjem.119.85

11. Bihoreau MT, Dumas ME, Lathrop M, Gauguier D (2017) Genomic regulation of type 2 diabetes endophenotypes: contribution from genetic studies in the Goto-Kakizaki rat. Biochimie 143: 56-65. https://doi.org/10.1016/j.biochi.2017.08.012

12. Calderari S, Ria M, Gérard C et al (2017) Molecular genetics of the transcription factor GLIS3 identifies its dual function in beta cells and neurons. Genomics 110(2):98-111. https://doi.org/10.1016/j. ygeno.2017.09.001

13. Trung VN, Yamamoto H, Yamaguchi T et al (2013) Effect of sleeve gastrectomy on body weight, food intake, glucose tolerance, and metabolic hormone level in two different rat models: GotoKakizaki and diet-induced obese rat. J Surg Res 185(1):159-165. https://doi.org/10.1016/j.jss.2013.05.019

14. Saeidi N, Meoli L, Nestoridi E et al (2013) Reprogramming of intestinal glucose metabolism and glycemic control in rats after gastric bypass. Science 341(6144):406-410. https://doi.org/10. 1126/science. 1235103

15. Salinari S, le Roux CW, Bertuzzi A, Rubino F, Mingrone G (2014) Duodenal-jejunal bypass and jejunectomy improve insulin sensitivity in Goto-Kakizaki diabetic rats without changes in incretins or insulin secretion. Diabetes 63(3):1069-1078. https://doi.org/10. 2337/db13-0856

16. Manichanh C, Reeder J, Gibert P et al (2010) Reshaping the gut microbiome with bacterial transplantation and antibiotic intake. Genome Res 20(10):1411-1419. https://doi.org/10.1101/gr. 107987.110

17. Bartolí R, Boix J, Odena G, de Vega MV, Lorenzo-Zúñiga V (2012) Determination of the ideal preparation for colonoscopy in a rat model. Surg Laparosc Endosc Percutan Tech 22(6):542-545. https://doi.org/10.1097/SLE.0b013e318264c4f9

18. Brial F, Le Lay A, Hedjazi L et al (2019) Systems genetics of hepatic metabolome reveals octopamine as a target for nonalcoholic fatty liver disease treatment. Sci Rep 9(1):3656. https:// doi.org/10.1038/s41598-019-40153-0

19. Sarafian MH, Lewis MR, Pechlivanis A et al (2015) Bile acid profiling and quantification in biofluids using ultra-performance liquid chromatography tandem mass spectrometry. Anal Chem 87(19):9662-9670. https://doi.org/10.1021/acs.analchem.5b01556

20. Benjamini Y, Hochberg Y (1995) Controlling the false discovery rate: a practical and powerful approach to multiple testing. J R Stat Soc 57(1):289-300

21. Pedersen HK, Gudmundsdottir V, Nielsen HB et al (2016) Human gut microbes impact host serum metabolome and insulin sensitivity. Nature 535(7612):376-381. https://doi.org/10.1038/nature18646

22. Kovatcheva-Datchary P, Nilsson A, Akrami R et al (2015) Dietary fiber-induced improvement in glucose metabolism is associated with increased abundance of Prevotella. Cell Metab 22(6):971982. https://doi.org/10.1016/j.cmet.2015.10.001

23. Rautio M, Lönnroth M, Saxén H, Nikku R, Väisänen M-L (1997) Characteristics of an unusual anaerobic pigmented gram-negative rod isolated from normal and inflamed appendices. Clin Infect Dis 25(Suppl 2):S107-S110. https://doi.org/10.1086/516210

24. Bozadjieva N, Heppner KM, Seeley RJ (2018) Targeting FXR and FGF19 to treat metabolic diseases - lessons learned from bariatric surgery. Diabetes 67(9):1720-1728. https://doi.org/10.2337/dbi170007 
25. Chiang JY (2013) Bile acid metabolism and signaling. Compr Physiol 3(3):1191-1212. https://doi.org/10.1002/cphy.c120023

26. Teodoro JS, Rolo AP, Palmeira CM (2011) Hepatic FXR: key regulator of whole-body energy metabolism. Trends Endocrinol Metab 22(11):458-466. https://doi.org/10.1016/j.tem.2011.07.002

27. Fang S, Suh JM, Reilly SM et al (2015) Intestinal FXR agonism promotes adipose tissue browning and reduces obesity and insulin resistance. Nat Med 21(2):159-165. https://doi.org/10.1038/nm. 3760

28. Labrecque J, Laforest S, Michaud A, Biertho L, Tchernof A (2017) Impact of bariatric surgery on white adipose tissue inflammation. Can J Diabetes 41(4):407-417. https://doi.org/10.1016/j.jcjd.2016. 12.003

29. Hallberg SJ, Gershuni VM, Hazbun TL, Athinarayanan SJ (2019) Reversing type 2 diabetes: a narrative review of the evidence. Nutrients 11(4). https://doi.org/10.3390/nu11040766

30. Le Chatelier E, Nielsen T, Qin J et al (2013) Richness of human gut microbiome correlates with metabolic markers. Nature 500(7464): 541-546. https://doi.org/10.1038/nature12506

31. Pallister T, Jackson MA, Martin TC et al (2017) Hippurate as a metabolomic marker of gut microbiome diversity: modulation by diet and relationship to metabolic syndrome. Sci Rep 7(1):13670. https://doi.org/10.1038/s41598-017-13722-4

32. Tremaroli V, Karlsson F, Werling M et al (2015) Roux-en-Y gastric bypass and vertical banded gastroplasty induce long-term changes on the human gut microbiome contributing to fat mass regulation. Cell Metab 22(2):228-238. https://doi.org/10.1016/j.cmet.2015.07. 009

33. Liou AP, Paziuk M, Luevano JM, Machineni S, Turnbaugh PJ, Kaplan LM (2013) Conserved shifts in the gut microbiota due to gastric bypass reduce host weight and adiposity. Sci Transl Med 5(178):178ra141. https://doi.org/10.1126/scitranslmed.3005687

34. Osto M, Abegg K, Bueter M, le Roux CW, Cani PD, Lutz TA (2013) Roux-en-Y gastric bypass surgery in rats alters gut microbiota profile along the intestine. Physiol Behav 119:92-96. https:// doi.org/10.1016/j.physbeh.2013.06.008

35. Arora T, Seyfried F, Docherty NG et al (2017) Diabetes-associated microbiota in fa/fa rats is modified by Roux-en- $\mathrm{Y}$ gastric bypass. ISME J 11(9):2035-2046. https://doi.org/10.1038/ismej.2017.70

36. Miyachi T, Nagao M, Shibata C et al (2016) Biliopancreatic limb plays an important role in metabolic improvement after duodenaljejunal bypass in a rat model of diabetes. Surgery 159(5):1360 1371. https://doi.org/10.1016/j.surg.2015.11.027

37. Tett A, Huang KD, Asnicar F et al (2019) The Prevotella copri complex comprises four distinct clades underrepresented in westernized populations. Cell Host Microbe 26(5):666-679.e667. https://doi.org/10.1016/j.chom.2019.08.018

38. David LA, Maurice CF, Carmody RN et al (2014) Diet rapidly and reproducibly alters the human gut microbiome. Nature 505(7484): 559-563. https://doi.org/10.1038/nature 12820
39. Wu GD, Chen J, Hoffmann C et al (2011) Linking long-term dietary patterns with gut microbial enterotypes. Science 334(6052):105108. https://doi.org/10.1126/science.1208344

40. De Filippo C, Cavalieri D, Di Paola M et al (2010) Impact of diet in shaping gut microbiota revealed by a comparative study in children from Europe and rural Africa. Proc Natl Acad Sci U S A 107(33): 14691-14696. https://doi.org/10.1073/pnas.1005963107

41. Leite AZ, Rodrigues NC, Gonzaga MI et al (2017) Detection of increased plasma interleukin-6 levels and prevalence of Prevotella copri and Bacteroides vulgatus in the feces of type 2 diabetes patients. Front Immunol 8:1107. https://doi.org/10.3389/fimmu. 2017.01107

42. Otto GW, Kaisaki PJ, Brial F et al (2019) Conserved properties of genetic architecture of renal and fat transcriptomes in rat models of insulin resistance. Dis Model Mech 12(7):dmm038539. https://doi. org/10.1242/dmm.038539

43. Albaugh VL, Banan B, Ajouz H, Abumrad NN, Flynn CR (2017) Bile acids and bariatric surgery. Mol Asp Med 56:75-89. https:// doi.org/10.1016/j.mam.2017.04.001

44. Jonker JW, Liddle C, Downes M (2012) FXR and PXR: potential therapeutic targets in cholestasis. J Steroid Biochem Mol Biol 130(3-5):147-158. https://doi.org/10.1016/j.jsbmb.2011.06.012

45. Ryan KK, Tremaroli V, Clemmensen C et al (2014) FXR is a molecular target for the effects of vertical sleeve gastrectomy. Nature 509(7499):183-188. https://doi.org/10.1038/nature13135

46. Raghow R, Yellaturu C, Deng X, Park EA, Elam MB (2008) SREBPs: the crossroads of physiological and pathological lipid homeostasis. Trends Endocrinol Metab 19(2):65-73. https://doi. org/10.1016/j.tem.2007.10.009

47. Xu X, So JS, Park JG, Lee AH (2013) Transcriptional control of hepatic lipid metabolism by SREBP and ChREBP. Semin Liver Dis 33(4):301-311. https://doi.org/10.1055/s-0033-1358523

48. Ramracheya RD, McCulloch LJ, Clark A et al (2016) PYYdependent restoration of impaired insulin and glucagon secretion in type 2 diabetes following Roux-En-Y gastric bypass surgery. Cell Rep 15(5):944-950. https://doi.org/10.1016/j.celrep.2016.03. 091

49. Dodd D, Mackie RI, Cann IK (2011) Xylan degradation, a metabolic property shared by rumen and human colonic Bacteroidetes. Mol Microbiol 79(2):292-304. https://doi.org/10.1111/j.13652958.2010.07473.x

50. Scher JU, Sczesnak A, Longman RS et al (2013) Expansion of intestinal Prevotella copri correlates with enhanced susceptibility to arthritis. Elife 2:e01202. https://doi.org/10.7554/eLife.01202

51. Wen C, Zheng Z, Shao T et al (2017) Quantitative metagenomics reveals unique gut microbiome biomarkers in ankylosing spondylitis. Genome Biol 18(1):142. https://doi.org/10.1186/s13059-017$1271-6$

Publisher's note Springer Nature remains neutral with regard to jurisdictional claims in published maps and institutional affiliations. 